\title{
Intelligent decision support in a nursing educational institution
}

\author{
Peter Kokol $^{1,2}$ \\ ${ }^{1}$ Faculty of Electrical Engineering, University of Maribor, Maribor, Slovenia \\ ${ }^{2}$ Faculty of Health Sciences, University of Maribor, Maribor, Slovenia
}

\section{Email address:}

Peter.kokol@um.si (P. Kokol)

\section{To cite this article:}

Peter Kokol. Intelligent Decision Support in a Nursing Educational Institution, American Journal of Nursing Science. Vol. 2, No. 2, 2013, pp. 14-17. doi: 10.11648/j.ajns.20130202.11

\begin{abstract}
Evidence-based management is defined as a process of translating best evidence into organizational management practices. Surprisingly only 15 percent of decisions are evidence based. In the paper we present the idea how intelligent systems can be used to improve the current situation and show in a case study how intelligent systems can be successfully used to extract evidence to improve management practices and decision making, especially in human resource management.
\end{abstract}

Keywords: Evidence Based Practice, Evidence Based Management, Decision Making, Intelligent Systems

\section{Introduction}

Executives routinely dose their organizations with strategic snake oil: discredited nostrums, partial remedies, or untested management miracle cure. In many cases, the facts about what works are out there - so why don't managers use them?

Harvard business review, January 2006

In spite of the fact that evidence best practice is a very popular slogan recently, many organizations still fail to implement practices that research has shown to be positively associated with firm or institution performance. Indeed, the gap between those who conduct research and those who should implement research findings into practice is enormously pervasive, especially in the field of health care, which is very surprising, because actually this field invented the evidence based movement (in the field of management, this movement is known as "evidence-based management" or EBM). In this paper we will first introduce the evidence based management and then a case study how intelligent systems can be used to extract evidence to improve management practices and resource management in a nursing educational institution.

\section{Decision Making and Evidence Based Management}

The idea of evidence based practice has actually evolved in the medical field. Dr. Sacket the individual most frequently associated with this idea defined the evidence based medicine as

The conscientious, explicit and judicious use of current best evidence in making decisions about the care of individual patients.

Surprisingly (Pffefer 2001) only 15 percent of decisions are evidence based. So on what do doctors rely instead of evidence? Bellow are five most frequented sources, namely:

- Obsolete knowledge gained in school

- Long standing but never proven traditions

- Pattern gleaned from experience

- They are using methods in which they believe are most skilled

- Information from hordes of vendors with products and services to sell

Managers are still more ignorant then doctors about which methods, tools, are most appropriate and they are even less eager to find that out using scientific paradigm. As Pfeffer (2001) emphasizes if doctors will practice medicine like managers there will be still more sick or death patients and many more doctors in jail. Most of seasoned practitioners either from medical or management field neglect to seek new evidence because they trust their own experiences, more than they trust research. Many managers also get companies in the trouble importing methods without regarding their specific environment, some managers use their own strengths as a basis of decision making (to 
hammer everything looks like a nail) and finally numerous decisions are driven by dogma and believe.

Formally based on the definition on evidence based medicine stated above evidence-based management is defined as a process of translating best evidence into organizational management practices. As introduced in (Rynes 2007) through evidence-based management, practicing managers should develop into experts who make organizational decisions informed by social science and organizational research-part of the zeitgeist moving professional decisions away from personal preference and unsystematic experience toward those based on the best available scientific evidence.

\subsection{Obstacles}

Not everything that can be counted counts, and not everything that counts can be counted.

Einstein

But not withstanding all advantages of evidence based management there are also some obstacles:

- There is too much evidence

- There is not enough good evidence

- The evidence does not apply

- $\quad$ People are trying to mislead

- You are trying to "mislead" yourself

- The site effects outweigh the cure

- $\quad$ Similarly to doctors the managers rely on following resources instead of evidence:

- Obsolete knowledge

- Personal experience

- $\quad$ Specialist skills

- Hype dogma

- Mimicry of top performers

\subsubsection{Intelligent Systems in Evidence Based Management and Decision Making}

Bakken (2001) among many others relates the evidence based practice with informatics and calls for actions in the informatics field. In this paper we present an even more advanced paradigm which could eventually help to further advance evidence based practice/management and may bridge some of above problems. It is a very hot topic in informatics and computer science and is called intelligent systems and is common practice in medicine, and health care in general.

Intelligent systems is a methodology for searching even large-scale databases for patterns; used mainly to find previously unknown relations between variables that may be useful and are leading to.

The evidence shows that it is best to start evidence to start your own intelligent system based evidence management on your own collected data or by your own company databases.

\section{Methods}

Usage of intelligent systems is not a rarity in modern data analysis anymore. The ability to track and evaluate every step in the decision making process is the most important factor for trusting the decisions gained with machine learning methods. This study uses one of the most suitable families of machine learning based data analysis techniques - i.e. decision trees. One of the main advantages of decision trees is their comprehensibility - i.e. the symbolic representation of the extracted knowledge. Additional to their possible use as classifiers, they represent a generalization of knowledge that is needed to differentiate between two or more output class values. Decision trees along with rule based classifiers are the only group of classifiers that perform classification by a sequence of simple, easy-to-understand tests whose semantics are intuitively clear to domain experts (Murthy 1998). Data analysis described in this paper was performed using libraries from Weka machine learning environment (Witten and Frank 2005). Two classical decision tree building techniques (J48 and SimpleCART) along with an advanced Alternating Decision Tree (ADTree) were used for building decision tree models. Although there are some more sophisticated decision tree implementations available in Weka, we selected three decision tree methods according to comprehensibility of the final decision tree.

$\mathrm{J} 48$ is an implementation of a decision tree technique that is based on $\mathrm{C} 4.5$ algorithm which was originally proposed by Quinlan (1986). C4.5 algorithm is an extension of Quinlan's previous ID3 (Iterative Dichotomiser 3) method. SimpleCart method is widely known by the name CART (Classification And Regression Tree) analysis which is based on the paper by Breiman et al. (1984). CART approach can also produce classification or regression trees, which depends on the type of the dependent variable (categorical or numerical). Both techniques are regarded as golden standard in decision trees for classification.

ADTree (Freund and Mason 1999) is an advanced decision tree building technique based on boosting (Schapire 1990) algorithms that are usually used when ensembles of classifiers (Dietterich 2001) are built. In this specific case, boosting is used to "boost" the extraction of knowledge in the form of separate branches in a decision tree. Therefore the complexity of decision tree interpretation is higher, but on the other hand ADTree also performs much better in terms of classification accuracy.

Performance for each of the decision trees was evaluated by measuring the classification accuracy (ACC) and Area under ROC Curve (AUC) metrics. Due to small sample size leave-one-out cross-validation technique was used, where single sample from the original dataset is used as the validation data, and the remaining observations as the training data. This procedure is repeated such that each sample in the dataset is used once as the validation data.4. Using the Template.

\section{Results}

This section presents the results from decision tree based data analysis. The study was conducted using results from a Job satisfaction survey that included 21 employees at Faculty of Health Sciences at University of Maribor. Because of 
the limited space, we present only one of the most interesting decision trees. Please note that Lickert scale from 1 to 5 was used, where 1 represented the most negative opinion and 5 the most positive.

Altogether 7 rules can be derived from the sample decision tree displayed in Figure 1. Accuracy of LOOCV for this tree is $72.73 \%$ with AUC at $68.38 \%$. One of the interesting rules includes 28th (»Would you like to attend additional education regarding your work? «) and 9th (»Are you satisfied with the look of your workplace?«) question. The rightmost rule tells us that positive answers contribute to more positive attitude of employees toward their satisfaction with their job in general. This rule supports Maslow's Theory of Motivation as it connects the outlook of workplace with the employee's willingness to learn new skills and consequently leads to better general satisfaction with the jobs.

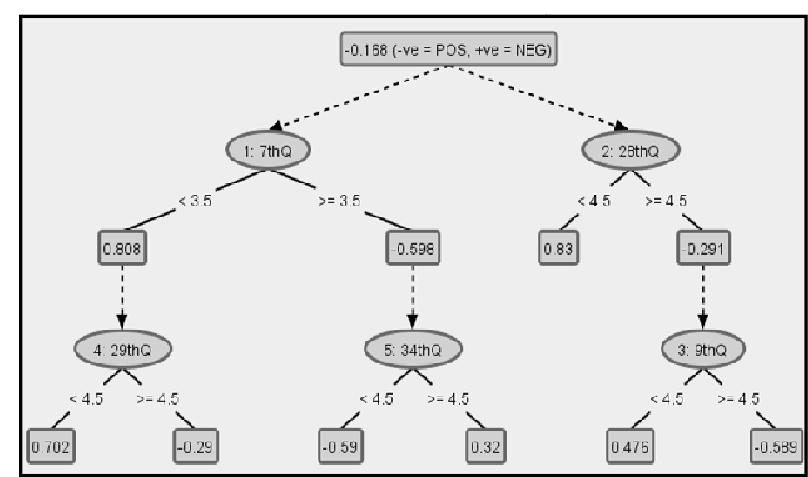

Figure 1. ADTree analysis of question: "Are you satisfied with your job in general? «.

It is also important to notice that access to information plays an important role in general satisfaction of employees (question 34). It is interesting that people that are not totally satisfied with the access to information (grade 4 or lower) are in general more satisfied with their job, compared to those that are perfectly satisfied with the access to information. However, this is only true for people that are satisfied with the collaboration between different units at the Faculty of Health Sciences. These two rules are new evidence, and maybe even a valuable new knowledge which can be used for better resource management. In practice this evidence could imply that in organizational units which have good communication with each other, to much information can lead to dissatisfaction - so for a middle level managers that could be a hint to not give too much information to their employs.

In Figure 2 an intelligent analysis of the difference between two groups of employees is presented. The first group represented academic staff and the second group represented administrative staff. In general the left branch in the Figure 2 is much more complex than the right one, where general satisfaction with the job is observed (question 4). On the left side one can observe the importance of 24th question asking employees whether they are satisfied with the award for their accomplished work. People answering with 4 or lower (mostly academic staff) are further separated on the basis of the question number 5. This question asked if they think their work contributes to the general successfulness of the Faculty of Health Sciences. It is interesting to note that most "Other" staff answered with 3 or lower to this question, which can be interpreted that at least some of them feel they cannot contribute to the successfulness of the faculty. On the other side of the tree, we can find employees that answered the question 24 with 5 and are further separated by their answers to question 15 (Satisfaction with co-workers relationship). The answers in this subgroup were strongly negative for the academic group and very positive for other staff.

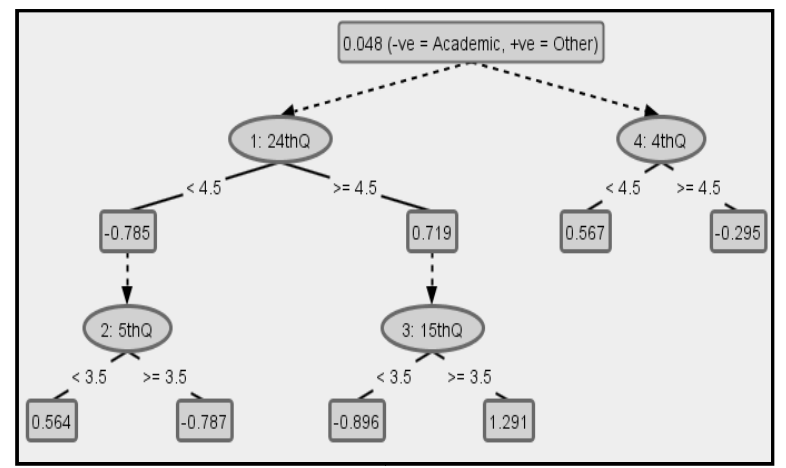

Figure 1. ADTree representing differences between "Academic" and "Other" staff.

\section{Conclusion}

In this paper we presented the strengths, weakness and obstacles of the evidence based management and how intelligent systems could be used to improve the situation. The results of the case study showed that the intelligent systems evidence based management paradigm can be very successful y to extract evidence and new knowledge and improve management.

\section{References}

[1] Bakken, S./McArthur, J. (2001): Evidence-based nursing practice: A call to action for nursing informatics, in: Journal of The American Medical Informatics Association, 8, 3, 289-290.

[2] Breiman, L./Friedman, J.H./Olshen, R.A./Stone, C.J. (1984): Classification and regression trees. Monterey, CA: Wadsworth, Inc.

[3] Dietterich, T.G. (2001): Ensemble methods in machine learning, in: Kittler, J., Roli, F. (eds.): Multiple Classifier Systems, LNCS Vol. 1857, Springer, 1-15.

[4] Murthy, S.K. (1998): Automatic construction of decision trees from data: A multi-disciplinary survey, in: Data Mining and Knowledge Discovery 2, 345-389.

[5] Pffefer, J., Sutton R.I. (2001), "Evidence Based Management”, Harward Business Review, jan 2008.

[6] Quinlan, J.R.: Induction of decision trees, in: Machine 
learning, 1 .

[7] Rynes S./Giluk T.L./Browm K.G. (2007): The very separate worlds of academia and practitioner periodicals in human resource management: Implications for evidence based management, in: Academy of Management Journal, 50, 5, 987-1008.

[8] Schapire R.E. (1990): The strength of weak learnability, in:
Machine Learning, 5, 2, 197-227.

[9] Witten, I.H./Frank, E.: Data Mining: Practical Machine Learning Tools and Techniques. Morgan Kaufmann Series in Data Management Systems, Morgan Kaufmann.

[10] Freund Y./Mason L. (1999): The Alternating Decision Tree Algorithm, in: Proceedings of the 16th International Conference on Machine Learning, 124-133. 